\title{
A comprehensive study of the genomic differentiation between temperate Dent and Flint maize
}

Sandra Unterseer ${ }^{1}$, Saurabh D. Pophaly ${ }^{2}$, Regina Peis ${ }^{1}$, Peter Westermeier ${ }^{1,3}$, Manfred Mayer ${ }^{1}$, Michael A. Seidel ${ }^{4}$, Georg Haberer ${ }^{4}$, Klaus F. X. Mayer ${ }^{4}$, Bernardo Ordas ${ }^{5}$, Hubert Pausch ${ }^{6}$, Aurélien Tellier ${ }^{2}$, Eva Bauer ${ }^{1}$ and Chris-Carolin Schön ${ }^{1 *}$

\begin{abstract}
Background: Dent and Flint represent two major germplasm pools exploited in maize breeding. Several traits differentiate the two pools, like cold tolerance, early vigor, and flowering time. A comparative investigation of their genomic architecture relevant for quantitative trait expression has not been reported so far. Understanding the genomic differences between germplasm pools may contribute to a better understanding of the complementarity in heterotic patterns exploited in hybrid breeding and of mechanisms involved in adaptation to different environments.

Results: We perform whole-genome screens for signatures of selection specific to temperate Dent and Flint maize by comparing high-density genotyping data of 70 American and European Dent and 66 European Flint inbred lines. We find $2.2 \%$ and $1.4 \%$ of the genes are under selective pressure, respectively, and identify candidate genes associated with agronomic traits known to differ between the two pools. Taking flowering time as an example for the differentiation between Dent and Flint, we investigate candidate genes involved in the flowering network by phenotypic analyses in a Dent-Flint introgression library and find that the Flint haplotypes of the candidates promote earlier flowering. Within the flowering network, the majority of Flint candidates are associated with endogenous pathways in contrast to Dent candidate genes, which are mainly involved in response to environmental factors like light and photoperiod. The diversity patterns of the candidates in a unique panel of more than 900 individuals from 38 European landraces indicate a major contribution of landraces from France, Germany, and Spain to the candidate gene diversity of the Flint elite lines.

Conclusions: In this study, we report the investigation of pool-specific differences between temperate Dent and Flint on a genome-wide scale. The identified candidate genes represent a promising source for the functional investigation of pool-specific haplotypes in different genetic backgrounds and for the evaluation of their potential for future crop improvement like the adaptation to specific environments.
\end{abstract}

Keywords: Maize, Flint, Dent, Selection, Population genetics, Genomics, Genome-wide screen, Landraces

\footnotetext{
* Correspondence: chris.schoen@tum.de

${ }^{1}$ Plant Breeding, TUM School of Life Sciences Weihenstephan, Technical

University of Munich, 85354 Freising, Germany

Full list of author information is available at the end of the article
} 


\section{Background}

Maize is one of the world's major staple crops but considerable concern is arising that ongoing anthropogenic global warming will have drastic effects on maize production and might result in a reduction of up to $10 \%$ in yield in the near future [1]. Expanding production areas to higher latitudes could moderate the effect, but this would require the adaptation of breeding material to shorter vegetation periods. Breeders can cope with this challenge by taking advantage of the tremendous genetic diversity of maize that is available in different temperate breeding pools. Two of the major pools exploited in breeding are the Dent and Flint germplasm pools with their names referring to different kernel phenotypes [2]. Dents have characteristic indented kernels with high soft starch content, whereas Flints have kernels with a thick, hard, and vitreous outer layer (Fig. 1a). The genetic divergence of these two pools can be explained by their historic geographical separation [3] and adaptation to different environments. Among all maize germplasm, Northern Flints reached the highest latitudes like the northern regions of the U.S. and Canada, which required selection for early maturity and cold tolerance [3]. These Northern Flints, together with Caribbean germplasm, were major progenitors of European maize and enabled the rapid adaptation to European climates [4]. Especially in cooler regions of Europe, breeding programs exploit heterotic effects between Dent lines tracing back to U.S. Corn Belt Dents and Flint lines, with Flint contributing early vigor and good cold tolerance and Dent contributing

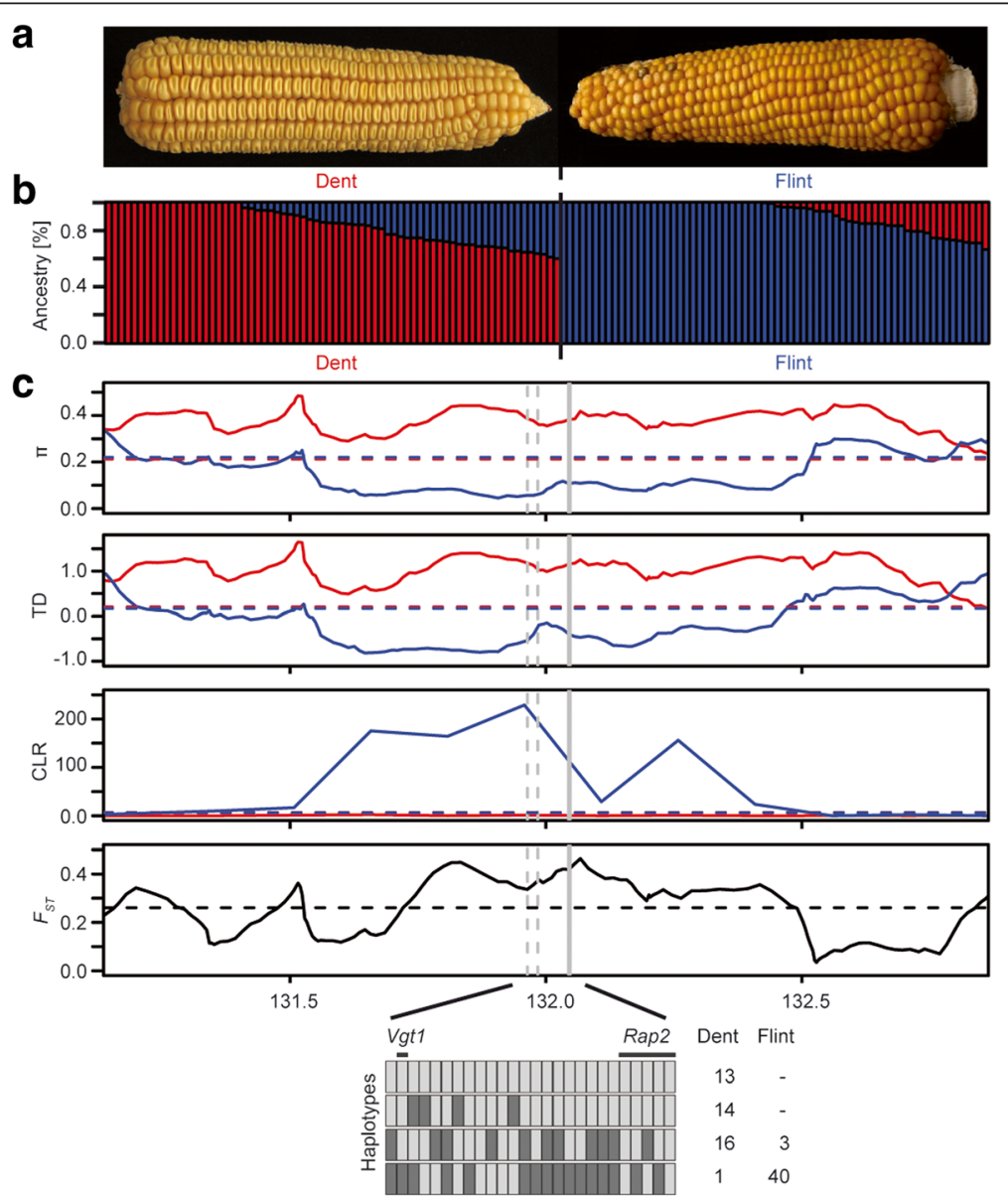

Fig. 1 Population structure of the investigated 136 Dent and Flint elite lines and detection of pool-specific selection signatures. a Images of maize cobs with Dent-type (left) and Flint-type kernels (right) as an example for phenotypic differences between the two germplasm pools. b Population structure and assignment of 136 temperate maize elite lines to Dent (red; $N=70)$ and Flint (blue; $N=66)$ pools. Bar plots indicate the relative ancestral composition of the lines. c Sweep statistics based on the panel of 136 temperate inbred lines shown exemplarily for a region on chromosome 8 that includes the Vgt1 locus (dashed gray lines) and Rap2 (solid gray line). Within-group statistics ( $\pi$, TD, and CLR) are shown in red for Dent and in blue for Flint. Horizontal dashed lines indicate the cutoff per statistic (10\% quantile for $\pi$ and TD, $90 \%$ quantile for CLR and $F_{S T}$ ). For the region encompassed by the two loci Vgt1 and Rap2, the four major haplotypes observed in the panel are shown. Light gray boxes indicate the B73 reference allele and dark gray boxes the alternative allele of each SNP. Numbers on the right side of the haplotype plot refer to the number of observations per haplotype within the Dent and the Flint panels 
high productivity to the hybrids. The divergence of the Dent and Flint germplasm pools has been described in diversity studies based on molecular markers [5] and also in genetic studies mapping quantitative trait loci (QTL) underlying agronomic traits. A recent study utilizing Dent and Flint nested association mapping (NAM) populations [6] found little overlap of QTL for five complex traits between the two pools [7]. Although QTL mapping is a useful tool to elucidate the genetic architecture of phenotypic traits, it can only unravel genomic regions for which the genetic material under study is segregating, whereas regions under selection can be missed in case of near or complete fixation. Thus, alternative approaches are needed to investigate the divergence of Dent and Flint on a genomic level and to further elucidate how selection shaped the pool-specific genomic diversity.

Selection creates specific patterns of diversity in the genome [8] and these signatures can be used for the detection of regions under selection. When a favorable, new (derived compared to the ancestral) allele rises in frequency within a population, selective sweeps are generated, which are characterized by a local reduction in nucleotide diversity and high derived allele frequencies [9-11]. In addition, strong and recent sweeps will display large blocks with high linkage disequilibrium surrounding the derived mutation as the dispersal of the new allele will be faster than recombination is able to break down linkage disequilibrium [12, 13]. The identification of selection signatures through genome-wide screens provides an efficient way to detect selection candidates and methods for their detection are often combined to reduce the number of false-positives [14-16]. In maize, genome-wide screens for selection signatures were successfully applied to identify genes involved in domestication and improvement and allowed insights into evolutionary processes shaping the genome diversity of maize [17-20]. Taking advantage of the characteristics of selective sweeps and using high-density genotyping data from a maize $600 \mathrm{k}$ single nucleotide polymorphism (SNP) array [21], we screened a panel of 136 temperate Dent and Flint elite lines for extreme allele frequencies over extended linked sites to identify genomic regions under selective pressure and to gain insights into the genomic variation underlying the differentiation of Dent and Flint. We included outgroup information from Sorghum bicolor to further support the identified candidate genes based on derived allele frequencies. We furthermore investigated the candidate genes based on whole-genome sequence data of 40 Dent and Flint lines $[21,22]$ and examined if genic and upstream regions contributed equally to the differentiation between temperate Dent and Flint.

The elite line panel under study comprised frequently used and important founder lines exploited in breeding programs for temperate climates. The Dent lines in our panel represent U.S. Corn Belt and European material, whereas most of the Flint lines originated from European breeding programs. Based on the selection screens, we examined pool-specific enrichment of candidate genes for metabolic pathways and investigated candidates associated with traits that are known to differentiate Dent and Flint like cold tolerance and flowering time [23, 24]. Flowering time is essential for local adaptation and represents a major determinant for other agronomic traits, such as grain filling and yield. The complex genetic architecture of flowering time has been studied in maize in a large number of studies mapping QTL with a meta-QTL analysis revealing 62 flowering time consensus QTL [25]. Phenotypic differences in maize flowering time are mainly caused by the accumulation of many small-effect QTL [26] and only a few large-effect genes have been characterized so far [27-30]. Hundreds of homologs to A. thaliana flowering time genes have been found in the maize genome [31], but in most cases their functional roles in the maize flowering network remain to be elucidated $[25,26$, 29, 32-37]. In this study, we identified candidate genes from the flowering network with haplotypes near fixation or fixed in either of the two elite pools. We used this set of genes as an example to characterize genomic differentiation between Dent and Flint in more detail. We evaluated the effect of these genes on flowering time in a Dent-Flint introgression library and investigated their assignment to different pathways within the flowering network. To assess the congruency of the allelic composition of the candidate genes between elite lines and landraces, we expanded our candidate gene analysis to a large dataset of 38 European landraces that comprises more than 900 individuals. By exploring this unique resource, we gained insights into the genetic variation of the selection candidates between landraces and elite lines and investigated, which landraces likely contributed to the observed candidate gene diversity in the elite lines and if haplotypes not yet exploited in breeding could be detected. Taken together, our study allowed insights into patterns of differentiation between temperate Dent and Flint germplasm and provided candidates for follow-up studies to characterize their biological and molecular functions, to investigate their impact on phenotypes, and to assess their potential use for further crop improvement.

\section{Results and discussion}

\section{Characterization of the Dent and Flint panels}

We genotyped a diverse panel of 136 temperate inbred lines (Additional file 1: Table S1) at high density with the Axiom $^{\circ}$ Maize Genotyping Array [21]. The array comprises more than $600 \mathrm{k}$ SNP markers, which were identified based on mid- to high-coverage whole-genome sequence data of 30 representative temperate Dent and 
Flint maize lines [21]. Markers were filtered according to quality scores and stable performance on the array, thus representing high-confidence sequence variants, and their final distribution followed the average recombination rate along the chromosomes [21]. After stringent quality filtering of the 616,201 markers included on the array, 547,412 high-quality SNPs (88.8 \%) remained for analysis. These SNPs tagged 19,759 genes ( $49.8 \%$ of the annotated gene set of maize) with, on average, two SNPs in their coding region (52.6 \% synonymous and $47.4 \%$ non-synonymous). Slightly more SNPs were polymorphic in the Flint compared to the Dent panel (95.4\% versus $93.1 \%$ ), but the majority of SNPs segregated in both germplasm pools (88.6\%).

The panel of 136 temperate Dent and Flint inbred lines comprised frequently used and important founder lines exploited in breeding programs in Europe and the U.S., including lines which were used as parents for the U.S. and European NAM panels [6, 38, 39]. The 70 Dent lines were selected according to available pedigree information and their frequency of use and citation [40, 41] to assemble a representative set of lines. Besides 16 European Dent lines, the lines represent U.S. Corn Belt Dent and include lines from the Maize Association Population [42] and the list of inbred lines with expired U.S. plant variety protection [43]. The 66 Flint lines investigated in this study comprised important founder lines of European breeding programs like F2 and F7 originating from the French landrace Lacaune, EP1 from the Spanish landrace Lizargarate, and derivatives of the German landrace Gelber Badischer Landmais [44]. The Flints comprised in total 34 lines from France, 20 from Germany, four from Spain, three from Italy, three from North America, as well as one from Switzerland and Austria. Between the elite lines of the two germplasm pools, we observed a clear separation of pools (Fig. 1b) and a high genome-wide level of differentiation $\left(F_{S T}=0.14\right)$, which is consistent with the long-term genetic differentiation between Dent-type and Flint-type maize $[2,3]$.

\section{Genome-wide screens for selection signals}

Taking advantage of the characteristics of selective sweeps, we screened the genome for extreme allele frequencies over extended linked sites to detect regions under differential selective pressure between Dent and Flint. Signatures of selection in only one of the two pools, Dent or Flint, were detected based on low levels of nucleotide diversity $(\pi)$ [9] and Tajima's $D$ (TD) [10] in the respective pool. In addition, a signature had to be supported by a high value of the composite likelihood ratio (CLR) test [11] within the respective pool, which indicates a deviation of the allelic composition of a genetic region compared to a neutrally evolving sequence determined by the genomic background. To ensure that the selection signature was specific for one of the two pools, it had to be associated with a high level of differentiation between Dent and Flint measured by the fixation index $F_{S T}$ [45]. Except for the CLR statistic, which was calculated for non-overlapping grids of $150 \mathrm{~kb}$, we applied a sliding window approach averaging data over windows of 40 SNPs (sliding by $10 \%$ ) and filtered for regions below the $10 \%$ quantile for $\pi$ and TD and above the $90 \%$ quantile for $F_{S T}$ and CLR (Additional file 1: Table S2). Following the approach reported by [17], adjacent windows passing the threshold for all four statistics were grouped together for candidate gene analysis, as the observed changes in allele frequency were likely caused by the same selective sweep event. This resulted in a filtered set of 265 windows for Dent and 158 windows for Flint, with an average length of $331.40 \mathrm{~kb}$ and $267.80 \mathrm{~kb}$, respectively, and thus comparable to the length of domestication windows found in a previous study [17]. An example of a signature of differential selection in Dent and Flint determined by all four metrics $\left(\pi, \mathrm{TD}, \mathrm{CLR}\right.$, and $F_{S T}$ ) is shown in Fig. 1c for a region on chromosome 8 harboring two candidate genes. The underlying genetic region was composed of four major haplotypes. The first three haplotypes occurred at intermediate frequencies in Dent, whereas the fourth haplotype was almost exclusive for Flint.

Genome-wide patterns of diversity and the resulting distribution of selection signatures in the Dent and Flint panels are given in Additional file 2: Figure S1. Within the filtered set of windows, which covered $4.3 \%$ of the total length of the maize genome for Dent and $2.1 \%$ for Flint, we identified 876 genes as candidates under differential selective pressure in Dent and 545 genes for Flint with 14 genes common to both candidate genes sets (Additional file 3: Table S3). This corresponded to $2.2 \%$ and $1.4 \%$ of the filtered gene set of maize, respectively, and is in the same order of magnitude as the estimated number of genes under selective pressure during maize domestication and improvement [17]. When comparing the candidate gene sets with the 571 improvement candidates reported by [17], 26 genes overlapped with the list of Dent candidates but only one gene with the Flint candidate gene set. Considering that the genetic material studied in [17] comprised mainly U.S. Dent and (sub-) tropical lines and that pool-specific sequence variation in temperate Dent and Flint has been reported here and, for example, by [5], these results emphasize the relevance of a representative panel of lines belonging to divergent germplasm pools to obtain a comprehensive picture of the genomic diversity in maize.

In genome-wide screens for signatures of positive selection, also other forces than selection, such as heterogeneous mutation and recombination rates along the genome, past demographic history and background 
selection shape the genomic diversity and can give rise to false-positive signals. It is beyond the scope of this paper to infer a full demographic history of maize for the elite lines and landraces as the breeding history of maize is complex and violates several assumptions of the classic population genetics models (e.g. discussed in [46]), as, for example, the assumption of panmictic populations and applicability of the coalescent at short time scales. We therefore applied the CLR test [11], which detects selective sweeps based on the comparison of the site-frequency spectrum within a specific genomic region to the average site-frequency spectrum over the genome, a method which has been successfully used in human and other species to detect selective sweeps $[11,47,48]$. To further decrease the rate of false-positives, the CLR test was combined with three additional metrics $\left(\pi, \mathrm{TD}\right.$, and $\left.F_{S T}\right)$ and we identified signatures of positive selection based on this conservative approach with an overlap of genomewide extreme values per metric. The high level of linkage disequilibrium in temperate Dent and Flint elite lines [21] facilitates the detection of selective sweep signals over sufficiently large genomic regions by the CLR test. On the other hand, the extent of linkage disequilibrium may decrease the power to discriminate between signals caused by genetic hitchhiking due to positive selection and negative background selection in regions with reduced levels of recombination $[49,50]$. To assess the number of falsepositives due to this effect, we explored the recombination landscape in the Dent and Flint panels by estimating lower bounds of historical recombination events [51]. The proportion of candidate genes located in regions with strongly reduced recombination rates and high linkage disequilibrium like (peri-) centromeric regions was then estimated. We found that $74.8 \%$ of the Dent and $80.9 \%$ of the Flint candidates were not located in regions with low levels of recombination (10\% quantile per chromosome; Additional file 1: Figure S2) indicating that the majority of candidates represent targets of selection rather than false-positive signals. Furthermore, in a classic selective sweep scenario (in contrast to background selection) targets of selection are to be enriched for derived alleles. As an additional test of our candidate regions, we included information from Sorghum bicolor to distinguish between ancestral and derived alleles. The Dent and Flint candidate gene sets revealed significantly higher derived allele frequencies compared to the remaining genes as measured by Fay and Wu's normalized $H$ [52] $(p<2.2 \mathrm{e}-16$; Additional file 1: Table S4), which also supported positive selection as the driving force of the observed allele frequency changes.

Gene ontology and pathway analyses of candidate gene sets Considering genetic differentiation and distinct phenotypic characteristics of Dent and Flint, we tested whether the candidate gene sets were enriched for specific biological processes or pathways. Gene ontology (GO) terms associated with the identified genes were available for around $40 \%$ of the candidates (333 for Dent and 214 for Flint). No significant GO term enrichment of biological processes, cellular components, and molecular functions could be detected for either of the two sets (Additional file 1: Figure S3). To investigate if candidate genes revealed a pool-specific enrichment for metabolic pathways, we performed pathway analyses using MapMan [53]. Based on information available for 58 Dent and 40 Flint candidate genes, we observed a grouping of genes associated with tetrapyrroles (chlorophyll and heme precursors) for Dent and for terpenoid metabolism for Flint (Additional file 1: Figure S4). The latter included the two genes ZmPPS7.3 (GRMZM2G014508) and ZmPPS8.2 (GRMZM2G483889), which encode a large and a small subunit of the geranyl diphosphate synthase complex in maize, respectively [54]. Like their homologues in A. thaliana [55], they are assumed to be involved in the biosynthesis of precursors of hormones from the isoprenoid pathway (e.g. gibberellins, brassinosteroids, and abscisic acid). The ability to produce other downstream products of this enzyme, namely $\beta$-caryophyllenes, has been shown to differ between European Flint and U.S. Dent lines and suggested that this defense response signal against herbivores was largely lost in temperate U.S. Dent $[56,57]$. The analysis of candidates associated with other traits that are known to differentiate Dent and Flint revealed six Flint candidates that, according to GO terms, are related to cold tolerance, a trait that is characteristic for temperate Flint [24]. For two of the candidates, differential expression upon exposure to chilling temperature has been reported in maize (GRMZM2G035584 [58] and GRMZM2G095562 [59]) as well as for the homologous gene of GRMZM2G139680 in rice [60]. The molecular and functional characterization of the identified candidate genes in maize and the investigation of differences between Dent and Flint in the regulation of phytohormone pathways or secondary metabolism may provide further insights in the adaptation of maize to different environments. Up to now, comprehensive RNA expression data across various developmental stages and tissues are mainly available for U.S. Dent lines like B73, which underlines the need for a better structural and functional genomic characterization of the Flint germplasm pool and its unique properties.

\section{Assessing the phenotypic effects of candidate genes on flowering time in a Dent-Flint introgression library}

In the genome-wide selection screens, we identified 18 candidates for Dent and 12 candidates for Flint, which could be assigned to the flowering pathway based on previous reports in maize, GO terms, and/or sequence 
homology to flowering genes characterized in other species [30, 32-34, 61, 62]. We focused exemplarily on candidate genes associated with the flowering network in maize as flowering time is an important agronomic trait that differentiates temperate Dent and Flint. However, functional studies of these genes in maize were available for only $30 \%$ of the candidates (Additional file 4: Table S5). Here, we investigated the effect of the flowering time candidate genes in more detail using a maize introgression library.

The introgression library had a Dent genetic background with introgressions from a Flint donor line and comprised 97 lines, which carried single Flint segments and covered in total $50.9 \%$ of the Flint donor genome $(1048.7 \mathrm{Mb})$ with a median length of the donor genome segment size of $10.6 \mathrm{Mb}$ (average: $30.8 \mathrm{Mb}$; Additional file 5: Table S6). We obtained phenotypic data for male and female flowering time based on a field experiment carried out at two locations in Germany. Heritabilities were $0.60\left(\mathrm{CI}_{0.95}=[0.40 ; 0.73]\right)$ and $0.51\left(\mathrm{CI}_{0.95}=[0.27\right.$; 0.67]) for male and female flowering time, respectively. Phenotypic differences between the Dent and Flint parent were larger for male than for female flowering time (23.2 and 17.8 days, respectively). Based on the least significant difference $(\alpha=0.05), 63$ (64.9\%; Fig. 2a) and 16 lines (16.5 \%; Fig. 2b) differed significantly from the recurrent Dent parent for male and female flowering time, respectively. Fifteen of these lines had significant effects for both male and female flowering time $(\alpha=0.05)$. When correcting for multiple testing $(\alpha=0.05 / 97)$, six lines $(6.2 \%)$ differed significantly for male and none for female flowering time.

Of the 97 lines, 22 carried a Flint introgression harboring one or several of the flowering time candidates identified in the selection screens (Additional file 5: Table S6). Fourteen of the 30 candidates were represented in these
22 introgression lines. Seven lines carried a segment with one or more of seven flowering time candidates identified in Flint and nine lines carried one or more of six flowering time candidates identified in Dent. Six lines carried a segment with a combination of Dent and Flint candidates. Although 75 lines did not carry one of the flowering time candidates identified in our selection screens, they may carry other flowering time genes with alleles differing between the Dent and the Flint parent of the introgression library. Lines carrying the Flint haplotype of a Flint candidate differed significantly from the 75 lines which did not carry one of the flowering time candidates from the selection screens (93.1 versus 96.1 days, $p$ value $=0.011$; Fig. 2 a) . For the lines which carried the Flint haplotype of a Dent selection candidate, this difference was not significant. The results indicate that in the genetic material under study, the Flint haplotypes of Flint candidates promoted flowering time more than the Flint haplotypes of Dent candidates.

Of the six lines with significant difference in male flowering compared to the Dent parent after correcting for multiple testing ( $\alpha=0.05 / 97$ ), two carried Flint haplotypes of Flint candidates and one a Flint haplotype of a Dent candidate. One of the lines included the well-characterized large-effect region comprising the ethylene-responsive transcription factor Rap2 (related to APETALA2 7, ZmRap2.7, Rap2, GRMZM2G700665) and its regulatory upstream locus Vgt1 [62], a major QTL for flowering time in maize $[29,30]$. The other line contained $Z c n 1$ (one of several members of the ZEA CENTRORADIALIS or TERMINAL FLOWER1 (TFL1)-like gene family [32]; also Phosphatidylethanolamine-binding protein1, Pebp1, GRMZM2G092008), for which so far only a moderate effect on flowering time was reported in maize [63]. This gene is related to TFL1 in A. thaliana [32], which is an

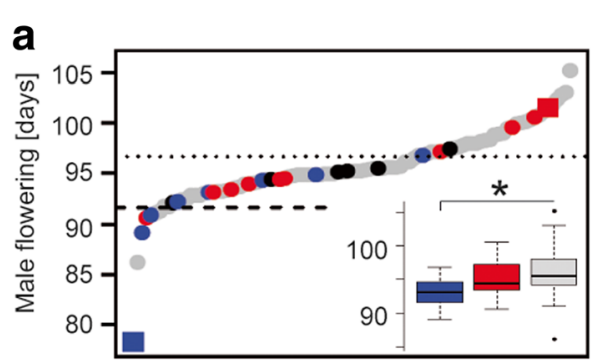

Introgression library lines

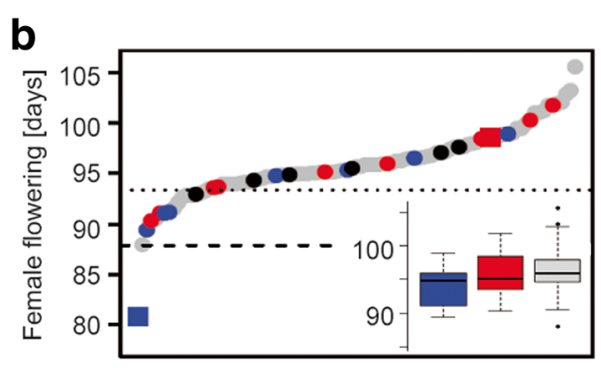

Introgression library lines

Fig. 2 Effect of candidate genes on flowering time in a Dent-Flint introgression library. Adjusted means of (a) male and (b) female flowering times for 97 introgression lines (circles) and the Dent and the Flint parental line (red and blue squares, respectively). Lines carrying a segment with Dent or Flint flowering time candidate genes are highlighted in red or blue, respectively, and lines with a Dent and a Flint candidate are shown in black. The dotted and dashed lines represent the significance thresholds without $(\alpha=0.05)$ and with correction for multiple testing $(a=0.05 / 97)$. Boxplots of adjusted means of flowering times are depicted in the lower parts of (a) and (b) for seven lines carrying Flint haplotypes of Flint flowering time candidates (blue), nine lines carrying Flint haplotypes of Dent flowering time candidates (red), and the 75 lines not carrying a flowering time candidate (gray). For details about the respective lines see Additional file 5: Table S6. Boxplots show the upper and lower quartile, median (horizontal bar), and whiskers (vertical bars) of the adjusted means. Points above and below the whiskers indicate values \pm 1.5 times the interquartile range. Significance of Student's $t$-tests with $p<0.05$ is indicated by * 
antagonist of the FLOWERING LOCUS T $(F T)[64,65]$ and required for the maintenance of an indeterminate inflorescence meristem identity and the regulation of flowering time in A. thaliana and maize [63-66]. The line with the Dent candidate carried Zmm22 (MADS-transcription factor 69, Mads69, GRMZM2G171650), which was recently reported to be associated with variation in flowering time in maize [67] and is considered a candidate for maize domestication and/or improvement $[68,69]$.

Overall, our findings in the introgression library support the relevance of the investigated genomic regions and their associated candidates for promoting flowering time and confirm the quantitative nature of flowering time in maize, determined by many genes with small effects [26] and only few genes with larger effects. Here, the effects of $Z c n 1$ and $Z m m 22$ were stronger than reported previously, which may be attributed to a stronger substitution effect when replacing a Dent haplotype with a Flint haplotype. We will target potential expression differences of flowering time candidates in the Dent-Flint introgression library in future studies to characterize possible differences in the regulation of the flowering network between germplasm pools adapted to different environments.

\section{Differential selection on components of the flowering network within temperate maize}

We investigated the 30 flowering time candidates with respect to their assignment to endogenous pathways and pathways regulated by environmental factors within the flowering network to determine if different components of the flowering network were under selective pressure in Dent and Flint, respectively. Within the flowering network, Flint candidates were involved predominantly in endogenous signaling, hormone-dependent, and developmental processes (10 of 12 candidates, $83.3 \%$ ), whereas the Dent candidates indicated a prevalence for response to environmental factors like light and photoperiod (12 of 18 candidates, $66.7 \%$; Fig. 3a, Additional file 4: Table S5). As described above, Flint candidates included the well-characterized Rap2/Vgt1 locus and Zcn1. Furthermore, we found the Squamosa promoter binding protein-transcription factor 25 (Sbp25, GRMZM2G414805) and Gnarley1 (Gn1; also Homeobox protein KNOTTED1-like 4, Knox4, GRMZM2G452178) that are associated with aging and hormone-dependent pathways (Additional file 4: Table S5). Gn1 is likely to act upstream of the "green revolution" gene encoding gibberellin 20-oxidase [70] and to regulate Gibberellin 2oxidase 1 expression in maize, thus influencing vegetative to reproductive phase transition, pollen tube growth, and stem elongation by changing the availability of gibberellin [71]. Gibberellin 2-oxidase 1 is additionally regulated by Knotted1 (Kn1, GRMZM2G017087) which

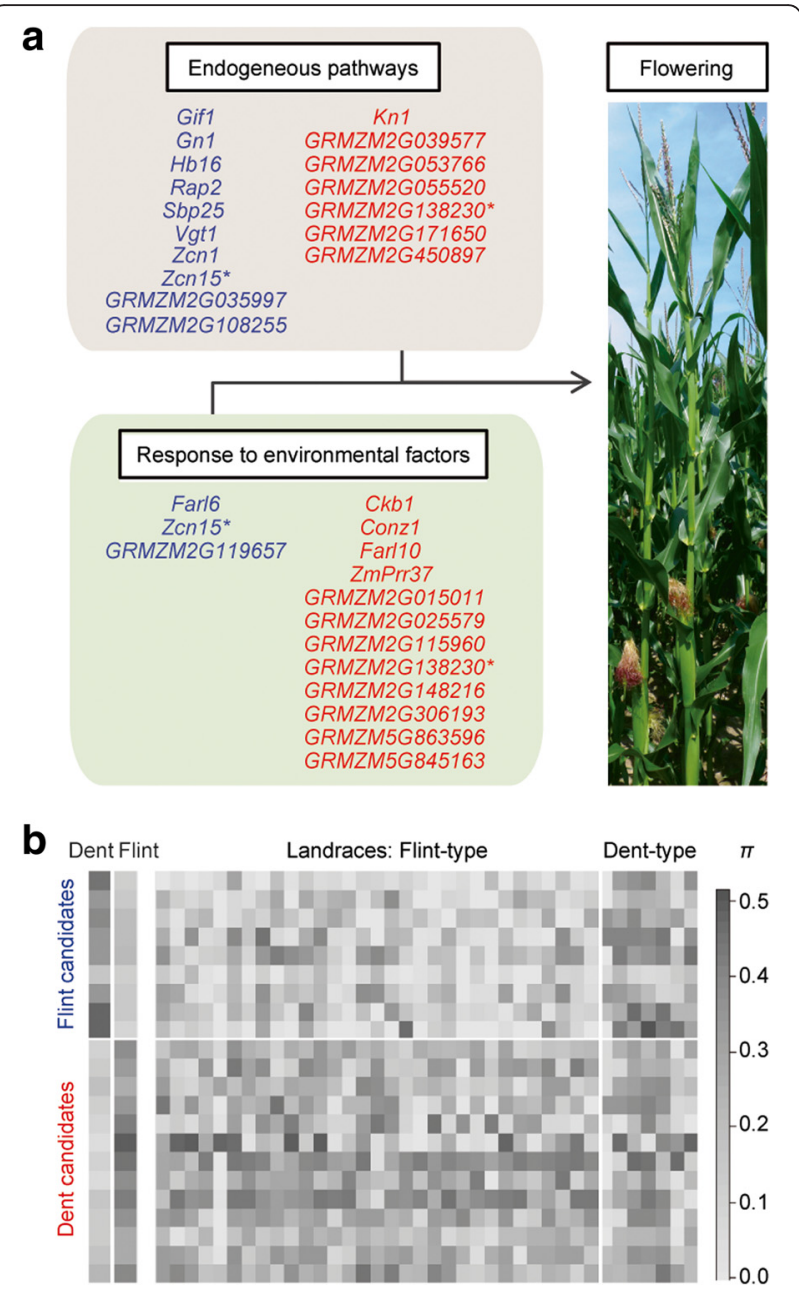

Fig. 3 Selection candidates of the maize flowering network and their nucleotide diversity in 136 elite lines and 38 European landraces. a Candidates associated with the maize flowering network identified under selective pressure in 70 Dent (red) and 66 Flint (blue) lines based on genotyping data. Candidates are grouped according to their putative function in endogenous pathways and pathways regulated by environmental factors. For details about the candidate genes and their classification, see Additional file 4: Table S5. Ambiguous assignments according to $\mathrm{GO}$ annotations and literature are indicated by ${ }^{*}$. $\mathbf{b}$ Nucleotide diversity $\pi$ of nine Flint (blue) and 13 Dent (red) flowering time candidate genes for 136 temperate elite lines as well as 31 Flint-type and seven Dent-type European landraces. Mean values for each gene were calculated for the panels of Dent and Flint elite lines (left) and for each of the 38 landraces (right). For details about candidate genes, gene-wise $\pi$ values, and order of landraces, see Additional file 6: Table S8

was identified as a Dent candidate gene [71]. Another well-characterized Dent candidate is Constans1 (Conz1, GRMZM2G405368), which is a putative ortholog of the photoperiod genes CONSTANS from $A$. thaliana and Heading date1 in rice [72]. To the best of our knowledge, 20 of the 30 detected flowering time candidates have not yet been functionally characterized in the context of maize flowering time, but were associated with 
the flowering network based on GO terms or reports in other species such as $A$. thaliana and rice (Additional file 4: Table S5). Thus, our study revealed candidates that warrant further investigation of their functional relevance in maize flowering time. Based on the observed allele frequency differences of the candidate genes within the 136 elite lines and with respect to their function in maize or, for example, A. thaliana, we hypothesize that different components of the flowering network were under selective pressure in Dent and Flint. The Flint-specific haplotypes of these genes might constitute a promising source for the adaptation of maize germplasm pools to shorter vegetation periods.

\section{Diversity of flowering time candidates in elite lines and European landraces}

As most of the European Flint inbred lines are assumed to be derived from few landraces [44], we compared the diversity and the allelic composition of 22 flowering time candidates (13 Dent and 9 Flint candidates tagged by at least five SNPs) between the elite lines and a unique panel of 38 European landraces (Additional file 1: Table S7). For each landrace, 22 to 24 plants were genotyped at high density with the Axiom ${ }^{\oplus}$ Maize Genotyping Array [21]. The majority of the landraces $(\mathrm{N}=31)$ had Flint-type kernels. These landraces exhibited lower levels of diversity in the Flint flowering time candidates (gene-wise average: $\pi=0.130)$ compared to the Dent flowering time candidates $(\pi=0.243)$, thus confirming the pattern found in the
Flint elite lines (Fig. 3b, Additional file 6: Table S8). We further investigated the level of differentiation between Flint-type landraces and Flint elite lines and observed low levels of $F_{S T}$ for the Flint flowering time candidates $\left(F_{S T}=0.060\right.$; Fig. $\left.4 \mathrm{a}, \mathrm{FT}\right)$ with ten landraces from France, Germany, and Spain displaying values even smaller than 0.050 (Additional file 7: Table S9). These low values of differentiation suggested a major contribution of the Flint-type landraces to the flowering time candidate gene diversity observed in the Flint elite lines. This hypothesis was corroborated by the finding that the entire set of Flint candidate genes also revealed significantly lower levels of differentiation compared to all other genes, which were not under differential selection between Dent and Flint elite lines $\left(F_{S T}=0.072\right.$ versus $0.095, p$ value $=6.0 \mathrm{e}-04$; Fig. $4 \mathrm{a}$, A versus $C$ ).

Consistent with the hypothesis that Flint elite lines and Flint-type landraces have a common history, significantly higher levels of differentiation were observed for Dent candidate genes compared to all remaining genes $\left(F_{S T}=0.111\right.$ versus $0.095, p$ value $=$ 0.017; Fig. 4b, A versus C). Together, these findings indicated that the reduced diversity observed for Flint candidate genes in Flint elite lines was already present in a broad panel of European landraces and that the candidate gene diversity of the Flint elite lines originate from a limited number of Flint-type landraces used for elite line development in some historically important breeding centers [44].
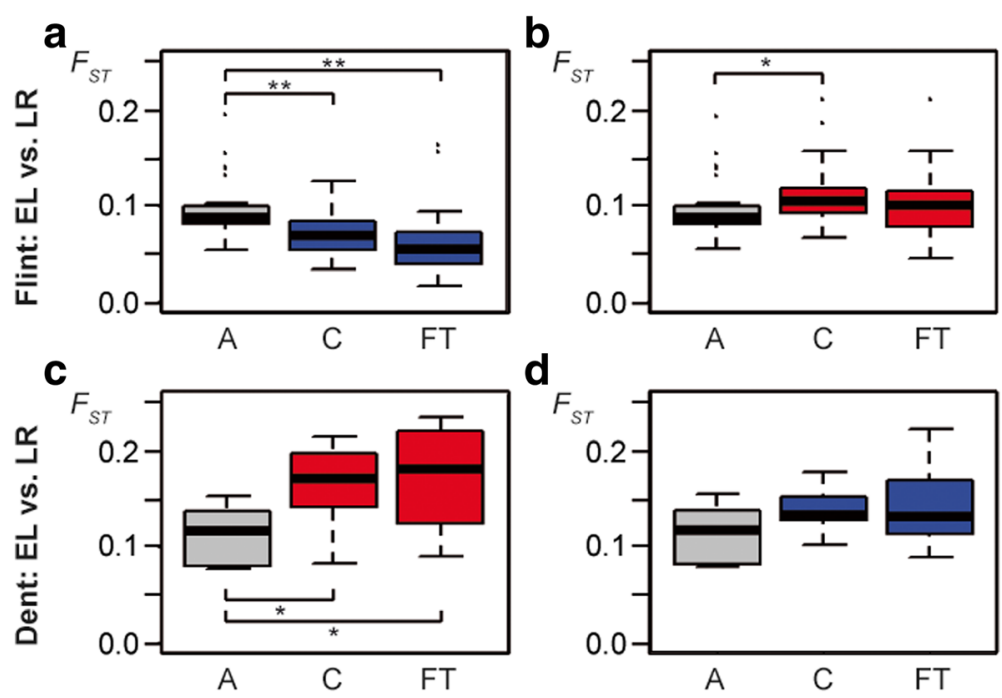

Fig. 4 Differentiation between elite lines (EL) and landraces (LR) for candidate genes. The upper panel shows the differentiation $\left(F_{S T}\right)$ between 66 Flint elite lines and 31 Flint-type landraces for (a) Flint (blue) and (b) Dent (red) candidate gene sets. The lower panel depicts the differentiation between 70 Dent elite lines and seven Dent-type landraces for (c) Dent (red) and (d) Flint (blue) candidate gene sets. The boxplots show $F_{S T}$ values for all (A; gray) genes except the candidates, the candidate (C) genes, and for the subset of candidates associated with flowering time (FT). Boxplots show the upper and lower quartile, median (horizontal bar), and whiskers (vertical bars) of the $F_{S T}$ values. Points above and below the whiskers indicate values \pm 1.5 times the interquartile range. Significance of two-sided Wilcoxon rank sum tests with $p<0.05$ are indicated by * and with $p<0.001$ by **. For details see Additional file 7: Table 59 
The remaining seven landraces displayed at least partially Dent-type kernels. These landraces revealed high levels of diversity for Dent and Flint flowering time candidates ( $\pi=0.225$ and 0.260 , respectively; Fig. 3b, Additional file 6: Table S8) and showed a high level of differentiation with Dent elite lines for the Dent flowering time candidates $\left(F_{S T}=0.170\right.$; Fig. $\left.4 \mathrm{c}, \mathrm{FT}\right)$. The same pattern was found in the analysis of the entire Dent candidate gene set, which revealed significantly higher levels of differentiation compared to all remaining genes $\left(F_{S T}=0.164\right.$ versus $0.111, p$ value $=0.026$; Fig. $4 \mathrm{c}$, A versus $C$ ), but no significant difference for Flint candidates compared to all remaining genes $\left(F_{S T}=0.138\right.$ versus $0.112, p$ value $=0.209$; Fig. $4 d$, A versus $C$; Additional file 7: Table S9). These results indicated that the European Dent-type landraces exhibit a different allelic composition in the Dent candidates compared to the Dent elite lines and did most likely not contribute to the Dent elite material under study.

\section{Selection on upstream and genic regions of the candidates}

To examine how specific elements of the genic regions contributed to the differentiation between Dent and Flint, we compared levels of differentiation for $5 \mathrm{~kb}$ and 500 bp upstream regions, genic regions, and exons between the candidate gene sets and all remaining genes. To increase the resolution of our analyses, we investigated the candidate gene sets based on whole-genome sequence data of 40 temperate elite lines (21 Dent and 19 Flint) [21, 22], which were part of the panel of 136 elite lines genotyped with the $600 \mathrm{k}$ array with the exception of three lines (Additional file 1: Table S1). Based on 13,246,294 bi-allelic SNPs, we observed a significant reduction of mean $\pi$ and TD in 727 Dent and 403 Flint candidate genes tagged by at least five SNPs (of in total 876 and 545 candidates, respectively) compared to 31,163 remaining genes ( $p$ value $<2.2 \mathrm{e}-16$; Additional file 1: Figure S5 and Additional file 1: Table $S 4) . F_{S T}$ values calculated between Dent and Flint were significantly higher for candidate gene sets compared to all remaining genes for $5 \mathrm{~kb}$ and $500 \mathrm{bp}$ upstream as well as genic and exonic regions. Together, these findings supported the results obtained from the selection screens in the panel of 136 temperate inbred lines genotyped with the $600 \mathrm{k}$ array.

Previous studies in maize suggested an important role of the divergence of regulatory elements in the context of domestication [73-75]. In our study, distributions of $F_{S T}$ values were comparable for $5 \mathrm{~kb}$ and $500 \mathrm{bp}$ upstream as well as genic and exonic regions in each of the two candidate gene sets (Additional file 1: Figure S6 and Additional file 1: Table S4). However, the power to resolve whether selection acted differentially in upstream and genic regions was probably limited by the high level of linkage disequilibrium observed in temperate Dent and Flint lines [21]. The outcome of ongoing large-scale whole genome and transcriptome sequencing will allow the investigation of the impact of selection on the regulation of gene activity in the two pools and its consequence for the genomic differentiation between Dent and Flint.

\section{Conclusions}

In this study, we report genomic differentiation between two major temperate maize germplasm pools, Dent and Flint. By comparing a representative panel of Dent and Flint elite lines, we identified candidate genes under differential selective pressure in Dent and Flint. The significant enrichment in derived allele frequencies for these genes provided strong indication that the candidate regions represented selective sweeps. Candidate genes associated with agronomic traits known to differ between Dent and Flint could be identified. Most of the detected flowering time candidates have not yet been functionally characterized in maize. Investigating the effect of the flowering time candidates in a Dent-Flint introgression library, we found that Flint haplotypes of these candidates promoted earlier flowering. Within the flowering network of maize, a Flint-specific enrichment of genes associated with endogenous signaling, hormonedependent pathways, and developmental processes was discovered in contrast to Dent, where selection seemed to act predominantly on genes involved in the response to environmental factors. Low levels of differentiation of Flint flowering time candidate genes between European Flint elite lines and European landraces indicated that the allelic composition of the elite lines was comparable to those of the Flint-type landraces and suggested a major contribution of landraces from France, Germany, and Spain to the candidate gene diversity in the Flint elite lines. Our findings highlight the role of genomic regions that have undergone intense selection and contributed to the differentiation of temperate Dent and Flint with likely effects on different agronomic traits. The identification of pool-specific selection signatures enabled insights into different patterns of diversity between temperate Dent and Flint and provides new targets for future functional analyses and crop improvement.

\section{Methods}

Plant material and genotyping of elite lines and landraces The 136 elite inbred lines (Additional file 1: Table S1) were selected to represent the genetic diversity of European and American temperate maize and were genotyped with the $600 \mathrm{k}^{\text {Affymetrix }}{ }^{\oplus}$ Axiom $^{\oplus}$ Maize Array described in [21]. Landraces were selected to reflect the genetic and phenotypic diversity within Central and Western Europe 
(Additional file 1: Table S7) and were represented by 22 to 24 plants each. All 906 landrace individuals were genotyped using the $600 \mathrm{k}$ array and the genotype cluster model file (http://www.affymetrix.com/catalog/ prod820010/AFFY/Axiom\%26\%23174\%3B-Maize-Genoty ping-Array).

If not denoted otherwise, analyses were performed using $\mathrm{R}$ version 3.0.1 [76]. SNP positions were assigned to the reference sequence B73 v2 [22] for all datasets. Analyses of the elite line panel were based on 566,961 best quality SNPs [21] with heterozygous calls masked as missing. Indels, unmapped markers, SNPs with $\geq 10 \%$ missing values in the elite line panel [21], markers designed to specifically differentiate between two Dent lines [77], and monomorphic SNPs were excluded, resulting in 547,412 SNPs for analyses of temperate elite lines. Analyses including the landraces were based on a subset of 486,208 SNPs.

\section{Population structure analysis and estimation of historical recombination rates}

For population structure analyses of the genotyped panel of 136 elite lines, missing data were imputed using Beagle [78] version 3.3.1 via the R package "synbreed" [79] version 0.10-3. A linkage disequilibrium pruning step was performed applying an $r^{2}$ threshold of 0.8 followed by the estimation of ancestry using ADMIXTURE [80] version 1.23. To obtain an estimate of the historical recombination rates in Dent and Flint based on the genotype data of the elite line panel, the four-gamete test [51] was calculated. This test gives a conservative estimate (i.e. the minimum number) of recombination events in the history of a sample. Values for the pairwise tests of neighboring SNPs were averaged over 1000 sites and the mean of each 1000 site bin was plotted. Reported were recombination events per $\mathrm{Mb}$ and regions of low recombination rates were defined as regions exhibiting rates within the $10 \%$ quantile per chromosome.

\section{Screens for selection signatures}

Nucleotide diversity $\pi$ [9] and Tajima's $D$ (TD) [10] were calculated for each panel of inbred lines using a customized script. The fixation index $F_{S T}$ [45] was calculated across the two panels using PLINK [81] version v1.90b2m. Metrics were calculated per SNP and averaged over windows of 40 SNPs (sliding by $10 \%$ and corresponding to an average physical distance of $10 \mathrm{~kb}$ ), using the R package "zoo" [82]. The grid-based composite likelihood ratio (CLR) test was calculated for each panel as implemented in SweepFinder [11]. For CLR, the size of the non-overlapping grids was $150 \mathrm{~kb}$, which is the same magnitude as the maximal distance between two SNPs with $r^{2}>0.2$ in the elite line panel [21]. Windows exhibiting values within the $10 \%$ quantile $(\pi, \mathrm{TD})$ and the $90 \%$ quantile $\left(F_{S T}, \mathrm{CLR}\right)$ were submitted for candidate gene analysis based on the B73 v2 [22] annotation, version $5 \mathrm{~b} 60$ (ftp://ftp.gramene.org/pub/gramene/ maizesequence.org/release-5b/filtered-set/), containing 39,656 gene models. GO terms were tested for enrichment using the resources developed by [83] based on maize gene IDs by applying a hypergeometric test with a Benjamini-Yekutieli correction ([84]; FDR $=0.05$ ) to account for multiple tests. Pathway analysis was performed using MapMan version 3.5.1 [53] based on the mapping of the first transcript of each gene to the file Zm_B73_5b_FGS_cds_2012.m02 downloaded from the MapMan webpage (http://mapman.gabipd.org/web/ guest/mapmanstore). For visualization, arbitrary values of -4.5 and 4.5 were assigned to Dent and Flint candidate gene transcripts, respectively.

\section{Whole-genome sequence datasets}

For Fay and Wu's normalized $H$ [52], the maizesorghum genome alignment (http://pipeline.lbl.gov/ downloads.shtml) was parsed with a custom Perl script to obtain the nucleotide in sorghum representing the ancestral maize allele for 298,388 SNPs of the genotyped panel of elite lines. Whole-genome sequence data for 30 elite lines were used to call SNPs and small indels by employing an integrative analysis pipeline [21] and filtering for high mapping $(\mathrm{MQ} \geq 30)$ and genotyping quality $(\mathrm{GQ}>5)$, major allele frequency $\geq 90 \%$, a minimal distance of 3 bp between adjacent SNPs, and a minimal and maximal coverage by three and $60 \mathrm{MQ} 30$ reads for lines sequenced to medium coverage, respectively. The latter criterion was adjusted for four deep sequenced lines requiring ten and $300 \mathrm{MQ} 30$ reads, respectively. We combined the obtained marker set with wholegenome sequence data from ten temperate inbred Dent and Flint lines from the maize HapMap2 project $[17,22]$ resulting in a VCF file including 13,246,294 bi-allelic SNPs. We filtered for SNPs with $\leq 50 \%$ missing values across the 40 lines $\left(F_{S T}\right)$ and within germplasm pools ( $\pi$ and TD). The combined VCF file was converted to hapmap format with a customized Perl script, and $\pi$ and TD per gene were obtained with Variscan version 2 [85] with runmode " 12 ".

For gene-wise calculations based on the genotyped panels of 136 elite lines and 38 European landraces (normalized $H, F_{S T}$ ) or on whole-genome sequence data of 40 elite lines ( $\pi$ and TD), the genic region of the longest protein-coding transcript, including $5 \mathrm{~kb}$ upstream, was used and metrics were calculated if at least 5 SNPs were available for analysis. For a separate analysis of exonic, genic, $500 \mathrm{bp}$, and $5 \mathrm{~kb}$ upstream regions, $F_{S T}$ was determined in case of at least 5 SNPs per region based on whole-genome sequence data using vcftools v0.1.11 [86]. Two-sided Wilcoxon rank sum tests [87] were 
performed on gene-wise metrics to test for differences between candidate genes and remaining genes within pools.

\section{Introgression library}

To represent the Flint genome as introgression segments in a Dent genetic background, a European Dent inbred line was crossed with a European Flint inbred line, followed by backcrossing, marker-assisted selection, and several rounds of selfing [88]. The Dent parental line originated from south-eastern Europe and exhibits a high general combining ability for kernel yield. The Flint parental line was selected for high general combining ability for biomass yield and good performance in Central European climates. The introgression library used in the present study comprised 535 lines, with 97 lines carrying a single segment of the Flint parent. To estimate the length of individual donor genome fragments, the distance between markers on the respective donor genome fragment plus half the distance to the adjacent marker flanking the donor genome fragment on either side of the fragment was calculated. For the introgression lines, the two parental lines, and a check, male and female flowering times were obtained from two field experiments conducted in 2014 at the German trial loca-

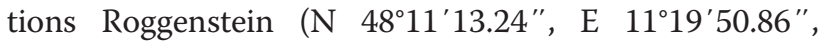
$517 \mathrm{~m}$ AMSL, average temperature in 2014: $9.8^{\circ} \mathrm{C}$ ) and Freising (N 48²4'11.62", E 1143'21.99", 480 m AMSL, average temperature in 2014: $9.7^{\circ} \mathrm{C}$ ). Each experiment was laid out as an $\alpha$-lattice design with two replications, except for parental lines and the check that were repeated three and five times, respectively. Male and female flowering time was recorded as days after sowing until $50 \%$ of plants per plot exhibited emerged anthers and silks, respectively. Adjusted means for flowering time were calculated using Plabstat [89]. The difference in adjusted means between the 97 single-segment introgression lines and the Dent parental line was tested at a significance level of $\alpha=0.05$ and $\alpha=0.05 / 97$ to correct for multiple testing. Adjusted means of flowering times for seven lines carrying Flint haplotypes of Flint flowering time candidates, nine lines carrying Flint haplotypes of Dent flowering time candidates, and the 75 lines not carrying a flowering time candidate gene were tested for significant differences by calculating Student's $t$-test.

\section{Additional files}

Additional file 1: Figure S2. Variation of historical recombination rates along the ten maize chromosomes. Figure S3. Gene ontology (GO) terms assigned to categories of biological processes for the candidate gene sets. Figure S4. Pathway analysis for the Dent and Flint candidate gene sets using MapMan [53]. Figure S5. Gene-wise $\pi$ (left) and TD (right) values based on whole-genome sequence data of 21 temperate Dent and 19 temperate Flint lines. Figure S6. Distributions of $F_{S T}$ values for different genomic regions based on whole-genome sequence data of 40 elite lines. Table S1. Elite lines under study with germplasm pool assignment and data source. Table S2. Window-based statistics and thresholds according to the selected quantile based on the panel of 136 temperate inbred lines genotyped with the $600 \mathrm{k}$ array. Table S4. Gene-wise statistics for all genes and for Dent and Flint candidate genes. Table S7. Landraces under study with their geographic origins. (PDF $1175 \mathrm{~kb}$ )

Additional file 2: Figure S1. Metrics of the selection screens for 136 temperate inbred lines along the ten maize chromosomes based on genotyping data. (PDF $4242 \mathrm{~kb}$ )

Additional file 3: Table S3. Gene-wise statistics for 1407 candidate genes identified in the 70 Dent $(N=876)$ and 66 Flint $(N=545)$ lines based on genotyping data. (XLSX $1238 \mathrm{~kb}$ )

Additional file 4: Table S5. Pathway assignment and information about candidates associated with the flowering network in maize. (XLSX 50 kb)

Additional file 5: Table S6. Genomic composition and flowering dates of 97 single-segment introgression library lines. (XLSX $114 \mathrm{~kb}$ )

Additional file 6: Table S8. Gene-wise nucleotide diversity $\pi$ and $F_{S T}$ for flowering time candidate genes with at least 5 SNPs in the genic and $5 \mathrm{~kb}$ upstream region. (XLSX $24 \mathrm{~kb}$ )

Additional file 7: Table S9. Gene-wise level of differentiation of allele frequencies between landraces and Dent (left) and Flint (right) elite lines based on genotyping data. (XLSX $27 \mathrm{~kb}$ )

\section{Acknowledgements}

We are grateful to Angel Alvarez (CSIC, Estación Experimental de Aula Dei, Zaragoza, Spain), Alain Charcosset (INRA, UMR de Génétique Quantitative et Evolution, Gif-Sur-Yvette, France), Barbara Eder and Joachim Eder (Bavarian State Research Center, Institute for Crop Science and Plant Breeding, Freising, Germany), Albrecht E. Melchinger (University of Hohenheim, Institute of Plant Breeding, Seed Science and Population Genetics, Stuttgart, Germany), Mark J. Millard (USDA-ARS, Ames, USA), Jesús Moreno González (CIAM, A Coruña, Spain), Rita Redaelli (CRA-MAC Maize Research Unit, Bergamo, Italy), Pedro Revilla (CSIC, Pontevedra, Spain), and Anne Zanetto (INRA, UE de Melgueil DiaScope, Mauguio, France) for providing elite line and/or landrace seeds. The original introgression library was kindly provided by Milena Ouzunova and Thomas Presterl (KWS SAAT SE, Einbeck, Germany). We thank Manfred Schönleben for his help with field trials and their analysis and Stefan Schwertfirm for his technical assistance, as well as Ruedi Fries (Technical University of Munich, Animal Breeding, Freising, Germany) for providing genotyping facilities. Furthermore, we thank our colleagues from the University of Hohenheim for kindly providing pictures of maize cobs and Joachim Hermisson (University of Vienna, Mathematics and BioSciences Group, Vienna, Austria) for fruitful discussions and helpful remarks during a research stay of SU in Vienna. We thank three anonymous reviewers for their valuable comments and suggestions.

\section{Funding}

This study was funded by the Federal Ministry of Education and Research (BMBF, Germany) within the AgroClustEr Synbreed - Synergistic plant and animal breeding (grant 0315528) and by the Spanish Ministry of Economy and Competitiveness (project RF2011-00022-C02-01 "Regeneration and rationalization of the maize landraces from the Iberian Peninsula"). Bernardo Ordas acknowledges a grant from the program "Ramón y Cajal" of the Spanish Ministry of Economy and Competitiveness.

\section{Availability of data and materials}

Genotype calls for elite lines and landraces are available at NCBI dbSNP upon Build B149 under accession number ss1998449529 to ss1999065412 and ss1999065413 to ss1999681296, respectively. Whole-genome sequence data for 30 elite lines are available under accession number PRJNA260788 at the NCBI BioProject webpage and the combined and filtered VCF file of wholegenome sequence data for 30 elite lines and the ten lines from the maize HapMap2 project $[17,22]$ are available at NCBI dbSNP under accession number ss1999681297 to ss2012927636 (upon Build B149). All three datasets are available at https://dx.doi.org/10.6084/m9.figshare.3427040.v1. 


\section{Authors' contributions}

CCS, EB, AT, and SU conceived the study and discussed the results; SU investigated genotypic and outgroup data; SU and MM analyzed genotypic data of landraces; MAS, GH, and KFXM carried out variant calling; SP extracted ancestral allele information; RP, SP, and SU performed sequence analyses; PW conducted the introgression library experiments; SU and EB investigated the introgression library data; $\mathrm{BO}$ contributed part of the Spanish landrace data; HP performed genotyping of elite lines and landraces; SU drafted the manuscript; EB, CCS, and AT edited the manuscript; all authors read and approved the final manuscript.

\section{Competing interests}

The authors declare that they have no competing interests.

\section{Ethics approval and consent to participate}

Not applicable.

\begin{abstract}
Author details
${ }^{1}$ Plant Breeding, TUM School of Life Sciences Weihenstephan, Technical University of Munich, 85354 Freising, Germany. ${ }^{2}$ Section of Population Genetics, TUM School of Life Sciences Weihenstephan, Technical University of Munich, 85354 Freising, Germany. ${ }^{3}$ Present Address: Institute for Crop Science and Plant Breeding, Bavarian State Research Center, 85354 Freising, Germany. ${ }^{4}$ Plant Genome and System Biology, Helmholtz Zentrum München, 85764 Neuherberg, Germany. ${ }^{5}$ Misión Biológica de Galicia, Spanish National Research Council (CSIC), 36080 Pontevedra, Spain. ${ }^{6}$ Animal Breeding, TUM School of Life Sciences Weihenstephan, Technical University of Munich, 85354 Freising, Germany.
\end{abstract}

Received: 16 December 2015 Accepted: 15 June 2016

Published online: 08 July 2016

\section{References}

1. Lobell DB, Tebaldi C. Getting caught with our plants down: the risks of a global crop yield slowdown from climate trends in the next two decades. Environ Res Lett. 2014:9:074003.

2. Smith CW, Betrán J, Runge ECA. Corn: origin, history, technology, and production. 1st ed. Hoboken, NJ: John Wiley \& Sons Inc.; 2004.

3. Brown WL, Anderson E. The northern flint corn. Ann Mo Bot Gard 1947:34:1-28.

4. Rebourg C, Chastanet M, Gouesnard B, Welcker C, Dubreuil P, Charcosset A. Maize introduction into Europe: the history reviewed in the light of molecular data. Theor Appl Genet. 2003;106:895-903.

5. Dubreuil P, Dufour P, Krejci E, Causse M, deVienne D, Gallais A, et al. Organization of RFLP diversity among inbred lines of maize representing the most significant heterotic groups. Crop Sci. 1996;36:790-9.

6. Bauer E, Falque M, Walter H, Bauland C, Camisan C, Campo L, et al. Intraspecific variation of recombination rate in maize. Genome Biol. 2013;14:R103.

7. Giraud H, Lehermeier C, Bauer E, Falque M, Segura V, Bauland C, et al. Linkage disequilibrium with linkage analysis of multiline crosses reveals different multiallelic QTL for hybrid performance in the flint and dent heterotic groups of maize. Genetics. 2014;198:1717-34.

8. Nielsen R. Molecular signatures of natural selection. Annu Rev Genet. 2005;39:197-218.

9. Tajima F. Evolutionary relationship of DNA sequences in finite populations. Genetics. 1983;105:437-60.

10. Tajima F. Statistical-method for testing the neutral mutation hypothesis by DNA polymorphism. Genetics. 1989;123:585-95.

11. Nielsen R, Williamson S, Kim Y, Hubisz MJ, Clark AG, Bustamante C. Genomic scans for selective sweeps using SNP data. Genome Res. 2005;15:1566-75.

12. Sabeti PC, Reich DE, Higgins JM, Levine HZP, Richter DJ, Schaffner SF, et al Detecting recent positive selection in the human genome from haplotype structure. Nature. 2002;419:832-7.

13. Voight BF, Kudaravalli S, Wen $X Q$, Pritchard JK. A map of recent positive selection in the human genome. PLoS Biol. 2006;4:446-58.

14. Long Q, Rabanal FA, Meng D, Huber CD, Farlow A, Platzer A, et al. Massive genomic variation and strong selection in Arabidopsis thaliana lines from Sweden. Nat Genet. 2013:45:884-90.
15. Pickrell JK, Coop G, Novembre J, Kudaravalli S, Li JZ, Absher D, et al. Signals of recent positive selection in a worldwide sample of human populations. Genome Res. 2009;19:826-37.

16. Qanbari S, Gianola D, Hayes B, Schenkel F, Miller S, Moore S, et al. Application of site and haplotype-frequency based approaches for detecting selection signatures in cattle. BMC Genomics. 2011;12:318.

17. Hufford MB, Xu X, van Heerwaarden J, Pyhäjärvi T, Chia JM, Cartwright RA, et al. Comparative population genomics of maize domestication and improvement. Nat Genet. 2012;44:808-U118.

18. Jiao YP, Zhao HN, Ren LH, Song WB, Zeng B, Guo JJ, et al. Genomewide genetic changes during modern breeding of maize. Nat Genet. 2012;44:812-U124.

19. Vigouroux Y, McMullen M, Hittinger CT, Houchins K, Schulz L, Kresovich S, et al. Identifying genes of agronomic importance in maize by screening microsatellites for evidence of selection during domestication. Proc Natl Acad Sci U S A. 2002;99:9650-5.

20. Yamasaki M, Tenaillon MI, Bi IV, Schroeder SG, Sanchez-Villeda H, Doebley $J F$, et al. A large-scale screen for artificial selection in maize identifies candidate agronomic loci for domestication and crop improvement. Plant Cell. 2005;17:2859-72.

21. Unterseer S, Bauer E, Haberer G, Seidel M, Knaak C, Ouzunova M, et al. A powerful tool for genome analysis in maize: development and evaluation of the high density 600 k SNP genotyping array. BMC Genomics. 2014;15:823.

22. Chia JM, Song C, Bradbury PJ, Costich D, de Leon N, Doebley J, et al. Maize HapMap2 identifies extant variation from a genome in flux. Nat Genet. 2012:44:803-7.

23. Camus-Kulandaivelu L, Veyrieras JB, Madur D, Combes V, Fourmann M, Barraud S, et al. Maize adaptation to temperate climate: relationship between population structure and polymorphism in the Dwarf8 gene. Genetics. 2006;172:2449-63.

24. Revilla P, Rodríguez VM, Ordás A, Rincent R, Charcosset A, Giauffret C, et al. Cold tolerance in two large maize inbred panels adapted to European climates. Crop Sci. 2014;54:1981-91.

25. Chardon F, Virlon B, Moreau L, Falque M, Joets J, Decousset L, et al. Genetic architecture of flowering time in maize as inferred from quantitative trait loci meta-analysis and synteny conservation with the rice genome. Genetics. 2004;168:2169-85.

26. Buckler ES, Holland JB, Bradbury PJ, Acharya CB, Brown PJ, Browne $C$, et al. The genetic architecture of maize flowering time. Science. 2009;325:714-8.

27. Colasanti J, Yuan Z, Sundaresan V. The indeterminate gene encodes a zinc finger protein and regulates a leaf-generated signal required for the transition to flowering in maize. Cell. 1998;93:593-603.

28. Muszynski MG, Dam T, Li B, Shirbroun DM, Hou Z, Bruggemann E, et al. Delayed flowering 1 encodes a basic leucine zipper protein that mediates floral inductive signals at the shoot apex in maize. Plant Physiol. 2006;142:1523-36

29. Salvi S, Tuberosa R, Chiapparino E, Maccaferri M, Veillet S, van Beuningen L, et al. Toward positional cloning of Vgt1, a QTL controlling the transition from the vegetative to the reproductive phase in maize. Plant Mol Biol. 2002;48:601-13.

30. Vladutu C, McLaughlin J, Phillips RL. Fine mapping and characterization of linked quantitative trait loci involved in the transition of the maize apical meristem from vegetative to generative structures. Genetics. 1999;153:993-1007.

31. Chen C, DeClerck G, Tian F, Spooner W, McCouch S, Buckler E. PICARA, an analytical pipeline providing probabilistic inference about a priori candidates genes underlying genome-wide association QTL in plants. PLOS One. 2012;7:e46596.

32. Danilevskaya ON, Meng X, Hou Z, Ananiev EV, Simmons CR. A genomic and expression compendium of the expanded PEBP gene family from maize. Plant Physiol. 2008;146:250-64.

33. Dong Z, Danilevskaya O, Abadie T, Messina C, Coles N, Cooper M. A gene regulatory network model for floral transition of the shoot apex in maize and its dynamic modeling. PLoS One. 2012;7:e43450.

34. Ducrocq S, Madur D, Veyrieras JB, Camus-Kulandaivelu L, Kloiber-Maitz M, Presterl T, et al. Key impact of Vgt1 on flowering time adaptation in maize: evidence from association mapping and ecogeographical information. Genetics. 2008:178:2433-7.

35. Xu J, Liu Y, Liu J, Cao M, Wang J, Lan H, et al. The genetic architecture of flowering time and photoperiod sensitivity in maize as revealed by QTL review and meta analysis. J Integr Plant Biol. 2012;54:358-373. 
36. Romay MC, Millard MJ, Glaubitz JC, Peiffer JA, Swarts KL, Casstevens TM, et al. Comprehensive genotyping of the USA national maize inbred seed bank. Genome Biol. 2013;14:R55.

37. Castelletti S, Tuberosa R, Pindo M, Salvi S. A MITE transposon insertion is associated with differential methylation at the maize flowering time QTL Vgt1. G3 (Bethesda). 2014:4:805-12.

38. McMullen MD, Kresovich S, Villeda HS, Bradbury P, Li H, Sun Q, et al. Genetic properties of the maize nested association mapping population. Science. 2009:325:737-40

39. Lehermeier C, Krämer N, Bauer E, Bauland C, Camisan C, Campo L, et al. Usefulness of multiparental populations of maize (Zea mays L.) for genomebased prediction. Genetics. 2014;198:3-16

40. Mikel MA. Availability and analysis of proprietary dent corn inbred lines with expired US plant variety protection. Crop Sci. 2006;46:2555-60.

41. Mikel MA, Dudley JW. Evolution of North American dent corn from public to proprietary germplasm. Crop Sci. 2006;46:1193-205.

42. Flint-Garcia SA, Thuillet AC, Yu J, Pressoir G, Romero SM, Mitchell SE, et al. Maize association population: a high-resolution platform for quantitative trait locus dissection. Plant J. 2005:44:1054-64.

43. Nelson PT, Coles ND, Holland JB, Bubeck DM, Smith S, Goodman MM Molecular characterization of maize inbreds with expired US plant variety protection. Crop Sci. 2008;48:1673-85.

44. Barrière $Y$, Alber D, Dolstra O, Lapierre C, Motto M, Ordas A, et al. Past and prospects of forage maize breeding in Europe: I. History germplasm evolution and correlative agronomic changes. Maydica. 2006;51:435-49.

45. Weir BS, Cockerham CC. Estimating F-statistics for the analysis of population structure. Evolution. 1984:38:1358-70.

46. Parat F, Schwertfirm G, Rudolph U, Miedaner T, Korzun V, Bauer E, et al. Geography and end use drive the diversification of worldwide winter rye populations. Mol Ecol. 2016;25:500-14.

47. Qanbari S, Pausch $H_{\text {, Jansen }}$, Somel M, Strom TM, Fries R, et al. Classic selective sweeps revealed by massive sequencing in cattle. PLoS Genet. 2014;10:e1004148.

48. Horton MW, Hancock AM, Huang YS, Toomajian C, Atwell S, Auton A, et al. Genome-wide patterns of genetic variation in worldwide Arabidopsis thaliana accessions from the RegMap panel. Nat Genet. 2012:44:212-6.

49. Stephan W. Genetic hitchhiking versus background selection: the controversy and its implications. Philos Trans R Soc Lond B Biol Sci. 2010;365:1245-53.

50. Charlesworth B, Morgan MT, Charlesworth D. The effect of deleterious mutations on neutral molecular variation. Genetics. 1993;134:1289-303.

51. Hudson RR, Kaplan NL. Statistical properties of the number of recombination events in the history of a sample of DNA sequences. Genetics. 1985:111:147-64.

52. Zeng K, Fu YX, Shi SH, Wu Cl. Statistical tests for detecting positive selection by utilizing high-frequency variants. Genetics. 2006;174:1431-9.

53. Thimm O, Blasing $O$, Gibon $Y$, Nagel A, Meyer S, Kruger $P$, et al. MAPMAN: a user-driven tool to display genomics data sets onto diagrams of metabolic pathways and other biological processes. Plant J. 2004;37:914-39.

54. Zhou M, Zhang Q, Wang C, Chen L, Sun Z, Zhu X, et al. Characterization of genes involved in isoprenoid diphosphate biosynthesis in maize. J Plant Growth Regul. 2015:34:294-308.

55. van Schie CC, Ament K, Schmidt A, Lange T, Haring MA, Schuurink RC. Geranyl diphosphate synthase is required for biosynthesis of gibberellins. Plant J. 2007;52:752-62

56. Degen T, Dillmann C, Marion-Poll F, Turlings TC. High genetic variability of herbivore-induced volatile emission within a broad range of maize inbred lines. Plant Physiol. 2004;135:1928-38.

57. Kollner TG, Held M, Lenk C, Hiltpold I, Turlings TC, Gershenzon J, et al. A maize (E)-beta-caryophyllene synthase implicated in indirect defense responses against herbivores is not expressed in most American maize varieties. Plant Cell. 2008;20:482-94.

58. Trzcinska-Danielewicz J, Bilska A, Fronk J, Zielenkiewicz P, Jarochowska E, Roszczyk M, et al. Global analysis of gene expression in maize leaves treated with low temperature I. Moderate chilling $\left(14^{\circ} \mathrm{C}\right)$. Plant Sci. 2009; 177:648-58.

59. Sobkowiak A, Jonczyk M, Jarochowska E, Biecek P, Trzcinska-Danielewicz J, Leipner J, et al. Genome-wide transcriptomic analysis of response to low temperature reveals candidate genes determining divergent cold-sensitivity of maize inbred lines. Plant Mol Biol. 2014;85:317-31.
60. Yan SP, Zhang QY, Tang ZC, Su WA, Sun WN. Comparative proteomic analysis provides new insights into chilling stress responses in rice. Mol Cell Proteomics. 2006;5:484-96.

61. Hung HY, Shannon LM, Tian F, Bradbury PJ, Chen C, Flint-Garcia SA, et al. ZmCCT and the genetic basis of day-length adaptation underlying the postdomestication spread of maize. Proc Natl Acad Sci U S A. 2012;109: E1913-21.

62. Salvi S, Sponza G, Morgante M, Tomes D, Niu X, Fengler KA, et al. Conserved noncoding genomic sequences associated with a flowering-time quantitative trait locus in maize. Proc Natl Acad Sci U S A. 2007;104:11376-81.

63. Danilevskaya ON, Meng X, Ananiev EV. Concerted modification of flowering time and inflorescence architecture by ectopic expression of TFL 1-like genes in maize. Plant Physiol. 2010;153:238-51.

64. Kobayashi Y, Kaya H, Goto K, Iwabuchi M, Araki T. A pair of related genes with antagonistic roles in mediating flowering signals. Science. 1999;286:1960-2.

65. Koornneef M, Hanhart CJ, van der Veen JH. A genetic and physiological analysis of late flowering mutants in Arabidopsis thaliana. Mol Gen Genet. 1991;229:57-66.

66. Hanano S, Goto K. Arabidopsis TERMINAL FLOWER1 is involved in the regulation of flowering time and inflorescence development through transcriptional repression. Plant Cell. 2011;23:3172-84.

67. Hirsch CN, Foerster JM, Johnson JM, Sekhon RS, Muttoni G, Vaillancourt B, et al. Insights into the maize pan-genome and pan-transcriptome. Plant Cell. 2014;26:121-35

68. Briggs WH, McMullen MD, Gaut BS, Doebley J. Linkage mapping of domestication loci in a large maize teosinte backcross resource. Genetics. 2007:177:1915-28.

69. Zhao Q, Weber AL, McMullen MD, Guill K, Doebley J. MADS-box genes of maize: frequent targets of selection during domestication. Genet Res (Camb). 2011;93:65-75.

70. Sasaki A, Ashikari M, Ueguchi-Tanaka M, Itoh H, Nishimura A, Swapan D, et al. Green revolution: A mutant gibberellin-synthesis gene in rice - New insight into the rice variant that helped to avert famine over thirty years ago. Nature. 2002;416:701-2.

71. Bolduc N, Hake S. The maize transcription factor KNOTTED1 directly regulates the gibberellin catabolism gene ga20x1. Plant Cell. 2009;21:1647-58.

72. Miller TA, Muslin EH, Dorweiler JE. A maize CONSTANS-like gene, conz1, exhibits distinct diurnal expression patterns in varied photoperiods. Planta. 2008:227:1377-88

73. Wang RL, Stec A, Hey J, Lukens L, Doebley J. The limits of selection during maize domestication. Nature. 1999:398:236-9.

74. Wang H, Nussbaum-Wagler T, Li B, Zhao Q, Vigouroux Y, Faller M, et al. The origin of the naked grains of maize. Nature. 2005;436:714-9.

75. Lemmon ZH, Bukowski R, Sun Q, Doebley JF. The role of cis regulatory evolution in maize domestication. PLoS Genet. 2014;10:e1004745.

76. R Core Team. R: A language and environment for statistical computing. Vienna: R Foundation; 2013.

77. Ganal MW, Durstewitz G, Polley A, Berard A, Buckler ES, Charcosset A, et al. A large maize (Zea mays L.) SNP genotyping array: development and germplasm genotyping, and genetic mapping to compare with the B73 reference genome. PLoS One. 2011;6:e28334.

78. Browning BL, Browning SR. A unified approach to genotype imputation and haplotype-phase inference for large data sets of trios and unrelated individuals. Am J Hum Genet. 2009;84:210-23.

79. Wimmer V, Albrecht T, Auinger HJ, Schön C-C. Synbreed: a framework for the analysis of genomic prediction data using R. Bioinformatics. 2012:28:2086-7.

80. Alexander DH, Novembre J, Lange K. Fast model-based estimation of ancestry in unrelated individuals. Genome Res. 2009;19:1655-64.

81. Chang CC, Chow CC, Tellier LC, Vattikuti S, Purcell SM, Lee JJ. Secondgeneration PLINK: rising to the challenge of larger and richer datasets. Gigascience. 2015;4:7.

82. Zeileis A, Grothendieck G. zoo: S3 infrastructure for regular and irregular time series. J Stat Softw. 2005;14:1-27.

83. Du Z, Zhou X, Ling Y, Zhang Z, Su Z. agriGO: a GO analysis toolkit for the agricultural community. Nucleic Acids Res. 2010;38:W64-70.

84. Benjamini $Y$, Yekutieli $D$. The control of the false discovery rate in multiple testing under dependency. Ann Statist. 2001;29:1165-88.

85. Hutter S, Vilella AJ, Rozas J. Genome-wide DNA polymorphism analyses using VariScan. BMC Bioinformatics. 2006;7:409. 
86. Danecek P, Auton A, Abecasis G, Albers CA, Banks E, DePristo MA, et al. The variant call format and VCFtools. Bioinformatics. 2011;27:2156-8.

87. Wilcoxon F. Individual comparisons by ranking methods. Biometrics Bull. 1945;1:80-3.

88. Gresset $\mathrm{S}$, Westermeier P, Rademacher $\mathrm{S}$, Ouzunova M, Presterl T, Westhoff $P$, et al. Stable carbon isotope discrimination is under genetic control in the $\mathrm{C}_{4}$ species maize with several genomic regions influencing trait expression. Plant Physiol. 2014;164:131-43.

89. Utz HF. PLABSTAT - A computer program for statistical analysis of Plant Bred experiments. Version 3A. Stuttgart: Universität Hohenheim; 2011.

Submit your next manuscript to BioMed Central and we will help you at every step:

- We accept pre-submission inquiries

- Our selector tool helps you to find the most relevant journal

- We provide round the clock customer support

- Convenient online submission

- Thorough peer review

- Inclusion in PubMed and all major indexing services

- Maximum visibility for your research

Submit your manuscript at www.biomedcentral.com/submit 